\title{
Bolt Slippage Evaluation in Column-tree Steel Moment Connection on L/d
}

\author{
Kangmin Lee, Keunyeong Oh*, Jaehyuk So \\ Dept. of Architectural Engineering/Chungnam National University \\ 99, Daehak-ro, Yuseong-gu, Daejeon, Korea \\ leekm@cnu.ac.kr; sniper7608@naver.com; sojh778@naver.com
}

\begin{abstract}
The purpose of this paper was to evaluate the bolt slippage in column-tree steel moment connection. Column-tree connections are composed of a shop-welded and field-bolted steel structure. However, it is known for that the bolt slippage was occurred in columntree connection system. Therefore, the bolt slippage in column-tree connection was examined the effects on seismic performance. So, there are fabricated with that $600,500,400 \mathrm{~mm}$ depth of beam were attached to same column size, respectively. As a result, the maximum load obtained from column face was increasingly decreased when the depth of beam was smaller. Also, if it is possible to control the L/d, it would be determined the section and amount of bolt slippage.
\end{abstract}

Keywords: Column-tree connection, Bolt slippage, Moment resisting frame, Beam splice, Cyclic loading.

\section{Introduction}

The column-tree connection has been used to one type of beam-to-column connection within steel moment resisting frames in Korea and Japan. In column-tree connections with steel moment resisting frames, short stub beams are welded to the column in the shop, and then middle portion of the beams are bolted to the column-tree in the field. For this reason, the shop welding of the stub beams to the columns provides for high quality and economical welding as well as easy inspection. Also, it is known that the field bolting of beam splices results in the economy and ease of field erection. Therefore, the use of column-tree system can be more economical than the other structural system that requires field-welding.

Although the beam splices were designed with slip critical connection, some researchers introduced about the bolt slippage in beam splices under cyclic loads. When the beam splice in steel structures was designed with slip critical connection, the bolt slippage does not theoretically occur. Also, some researcher suggested that if bolt slippage occurs under controlled conditions during cyclic loads, in many cases, the bolt slippage can improve seismic performance.

Therefore, in this paper, the bolt slippage of beam splices in beam was examined the effects on seismic performance when panel zone in beam-to-column structures was designed strongly. Also, when depth of the beam was different, the bolt slippage was evaluated the effects on the ratio of beam length and beam depth (L/d).

\section{Test Program}

For evaluating the bolt slippage in beam splice of column-tree connection, three full-scale test specimens of columntree connections, designed and detailed according to the AISC Seismic Provisions (AISC 2010) and Korean Building Code (KBC 2009), were fabricated and tested. Quasi-static cyclic loading was applied to the specimens to evaluate the hysteretic behaviour of column-tree connection.

\subsection{Design of Specimens}

The base specimen was fabricated according to the construction practice in Korea, in which the backing bars and weld taps are not eliminated. Although these factors cause brittle fracture at the beam-to-column connection, these practices were frequently conducted in Korea. For designing three full-scale column-tree specimens, the steel members most widely used in Korea were examined. As a result, most steel structures used H-600x200 beam (similar to W24x9) and H-400x400 column (similar to W14x14). The L/d of base specimen was 12.5 (i.e. length of beam is 7,500 mm). The base specimen was called 'DCT-12.5'. For evaluating the effects on bolt slippage within different steel member, H-500x200 and H-400x200 members were chosen, and then column members of each specimen were identically applied with 'DCT-12.5'. Because this study was 
evaluated to effect of bolt slippage within beam splices. At this time, the ratios of beam length and beam depth (L/d) of each specimen were 15.0 and 18.75, respectively. The each specimen was called 'DCT-15.0' and 'DCT-18.75', respectively.

Also, as mentioned earlier, the panel zone was strongly reinforced because this paper did not consider the effect of column within beam-to-column connection. For this reason, the ratio of $V_{p, s p l i c e}$ (expected shear strength at beam splice) and $V_{p, p z}$ (expected shear strength at panel zone) was designed to be close to zero. However, panel zone of 'DCT-18.75' specimen does not reinforce because it is close to zero without reinforcing panel zone. In full-scale tests, high-strength bolts of F10TM20 were used to erect the beam splice. The size and details of the specimens in Fig. 1, Fig, 2, and Fig. 3, respectively, and Table 1 summarizes the properties of each specimen.

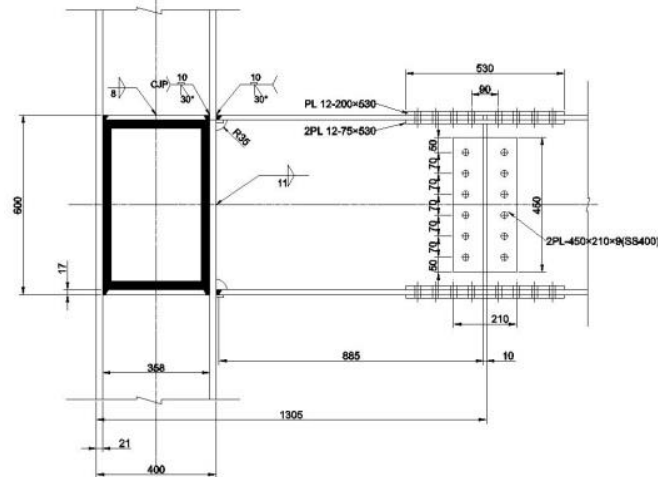

Fig. 1: Details of the 'DCT-12.5'.

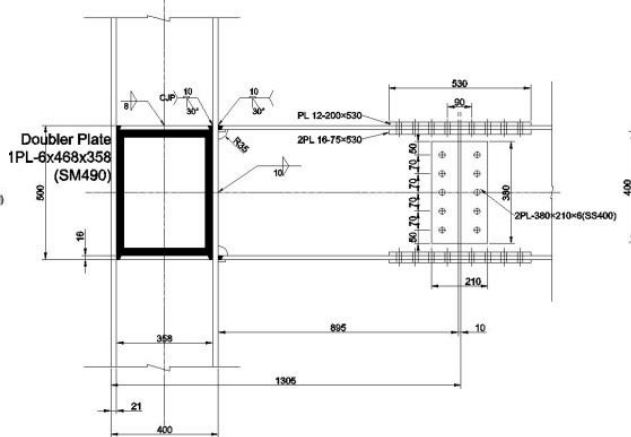

Fig. 2: Details of the 'DCT-15.0'.

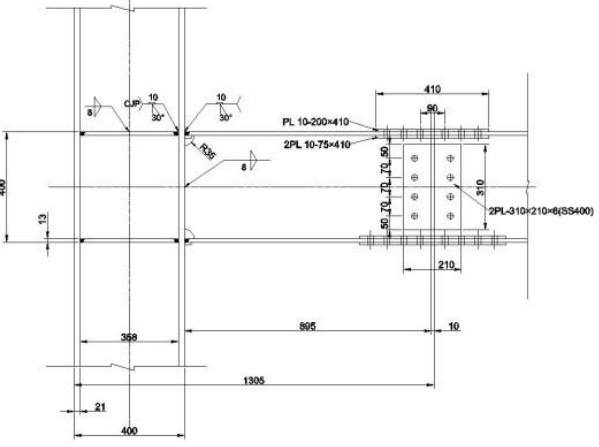

Fig. 3: Details of the 'DCT-18.75'.

Table 1: The properties of specimens.

\begin{tabular}{|c|c|c|c|c|c|c|c|c|}
\hline \multirow{2}{*}{ Specimen } & \multirow{2}{*}{$\begin{array}{c}\text { Column } \\
\text { size }(\mathrm{mm})\end{array}$} & \multirow{2}{*}{$\begin{array}{c}\text { Beam } \\
\text { size }(\mathrm{mm})\end{array}$} & \multicolumn{3}{|c|}{ Flange splice } & \multicolumn{2}{|c|}{ Web splice } & \multirow{2}{*}{$\mathrm{L} / \mathrm{d}$} \\
\hline & & & \multicolumn{2}{|c|}{ Flange plate $(\mathrm{mm})$} & Bots (EA) & Web plate $(\mathrm{mm})$ & Bots (EA) & \\
\hline \multirow{2}{*}{ DCT-12.5 } & \multirow{6}{*}{$\begin{array}{c}\mathrm{H}-400 \times 400 \\
\times 13 \times 21\end{array}$} & \multirow{2}{*}{$\begin{array}{c}\mathrm{H}-600 \times 200 \\
\times 11 \times 17\end{array}$} & Top & PL 12-530x200 & \multirow{2}{*}{32} & \multirow{2}{*}{ PL 9-450x210 } & \multirow{2}{*}{12} & \multirow{2}{*}{12.5} \\
\hline & & & Bot. & 2PL 12-530x75 & & & & \\
\hline \multirow{2}{*}{ DCT-15.0 } & & H-500x200 & Top & PL 12-530x200 & \multirow{2}{*}{32} & \multirow{2}{*}{ PL 6-380x210 } & \multirow{2}{*}{10} & \multirow{2}{*}{15.0} \\
\hline & & x10x16 & Bot. & 2PL 16-530x75 & & & & \\
\hline \multirow{2}{*}{ DCT-18.75 } & & H-400x200 & Top & PL 10-410x200 & \multirow{2}{*}{24} & \multirow{2}{*}{ PL 6-310x210 } & \multirow{2}{*}{8} & \multirow{2}{*}{18.75} \\
\hline & & x8x13 & Bot. & 2PL 12-410x75 & & & & \\
\hline
\end{tabular}

\subsection{Test Set-up and Loading History}

The test set-up was shown in Fig. 4. The test specimen consisted of a cantilever beam connected to a column flange. The column of each specimen was fixed to the strong floor, and the free end of beam was connected to the actuator with a loading capacity of $500 \mathrm{kN}$ (stroke $\pm 300 \mathrm{~mm}$ ). Also, guide frames were installed to prevent out-of-plane instability and twisting of the beam.

The specimens were tested by application of a prescribed quasi-static cyclic story drift ratio based on the loading protocol defined in Section S6.2 of the AISC Seismic Provision (AISC 2010). Story drift ratio was converted the displacement, and cyclic loading test was conducted by displacement control method. Two cycles of 5\% story drift ratio were added to the existing loading protocol. The loading protocol was shown in Fig. 5. 


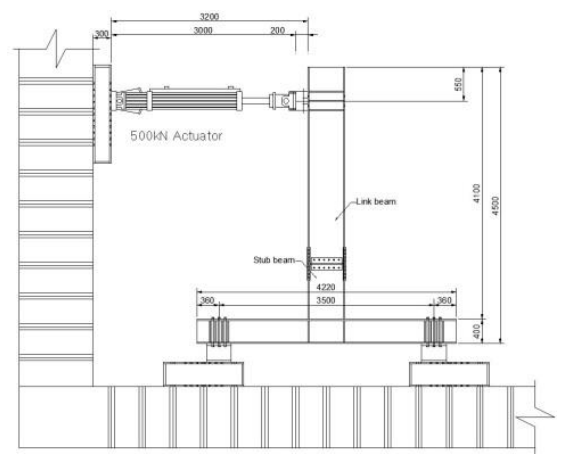

Fig. 4: Test set-up.

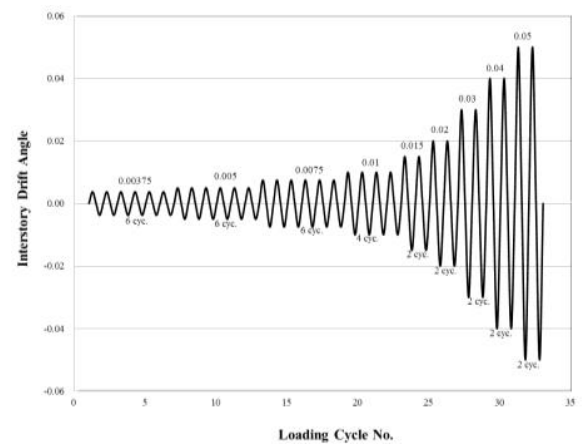

Fig. 5: Loading protocol.

\section{Test Results}

The tests were conducted until 5\% story drift ratio because of the limit of the actuator stroke and capacity. The normalized moment versus the story drift ratio relationships of the three full-scale specimens were shown in Fig. 6 . $M$ is the beam moment at the column face obtained from the test results, and $M_{p}$ is the beam plastic moment obtained from coupon test results.
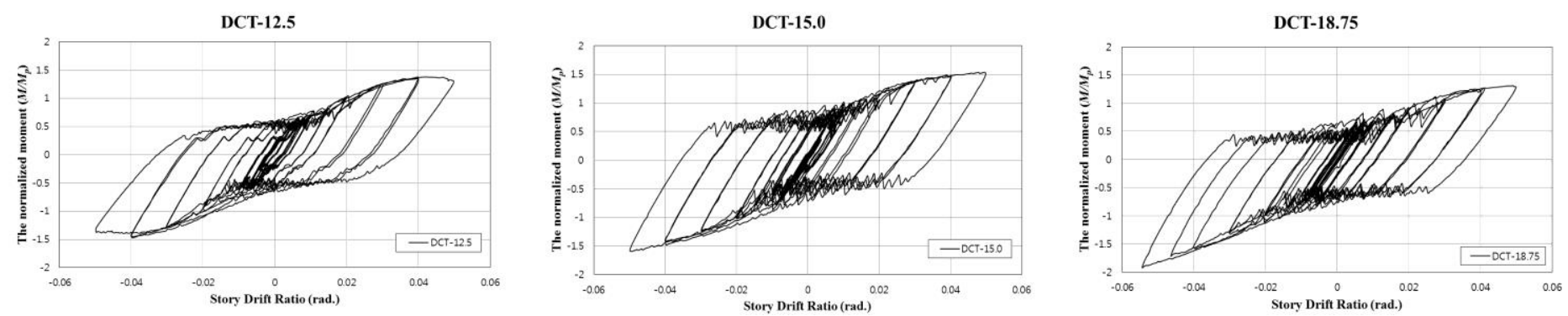

Fig. 6: The normalized moment-story drift ratio.

As seen in Fig. 6, the maximum moments of all specimens were about 1.0 times higher than the beam plastic moment. Also, three specimens developed an excellent connection cyclic rotation capacity up to 5\% story drift ratio without brittle fractures. For these reason, all specimens satisfied with the special moment frame (SMF) condition demanded by the AISC Seismic Provision and KBC 2009.

The smaller depth of beam was, the smaller the maximum moment of specimen was. Also, the range of bolt slippage was longer when the beam depth was shorter. A summary of the response of each specimen is given in Table 2, including maximum load, slip load, maximum displacement, normalized moment, plastic rotation, and final failure mode.

Table 2: Summary of test results.

\begin{tabular}{|l|c|c|c|c|c|c|}
\hline Specimen & $P_{\max }(\mathrm{kN})$ & $P_{s}(\mathrm{kN})$ & $\Delta_{\max }(\mathrm{mm})$ & $M / M_{p}$ & $\theta_{p}(\mathrm{rad})$ & Final failure mode \\
\hline DCT-12.5 & 256.9 & 105.4 & 187.0 & 1.38 & 0.0498 & Yielding \\
\hline DCT-15.0 & 209.7 & 117.5 & 186.9 & 1.54 & 0.0498 & Yielding \\
\hline DCT-18.75 & 109.3 & 68.6 & 187.0 & 1.31 & 0.0498 & Yielding \\
\hline
\end{tabular}

Note: $P_{\max }=$ maximum load, $P_{s}=$ average slip load, $\Delta_{\max }=$ maximum displacement, $M / M_{p}=$ normalized moment $\theta_{p}=$ total plastic rotation

\section{Analysis of Experimental Results}

The time of yielding and trend of deformation capacity for each specimen was checked. 'DCT-18.75' specimen was checked to develop more initial bolt slip than other specimens. 


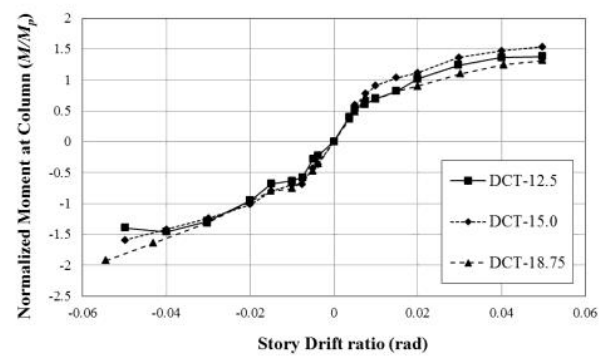

Fig. 7: Compared the trend of deformation capacity of each specimen.

The energy dissipation capacity is an important index to evaluate the seismic performance of beam-to-column moment connections, and it could be reflected through the area of load-displacement hysteretic curve. 'DCT-12.5' specimen with large beam depth dissipated the most amounts of energy, larger amounts than that dissipated by others.

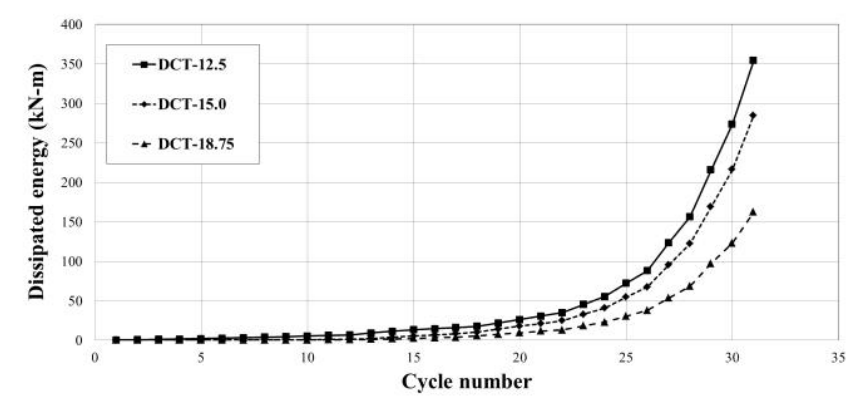

Fig. 7: Compared the trend of deformation capacity of each specimen.

\section{Conclusion}

Through this study, when the L/d was smaller, effects on bolt slippage were not affected within overall system. However, if it could be controlled the L/d, seismic performance and bolt slippage would be adjusted in beam-to-column connection.

\section{Acknowledgements}

This research was supported by Mid-career Researcher Program through National Research Foundation of Korea (NRF) funded by the Ministry of Science, ICT and Future Planning (grant number: 2015R1A2A2A01006765). Participants in the research were sponsored by the BK21 Plus project.

\section{References}

[1] AISC, Specification for structural steel buildings. American Institute of Steel Construction, 2010.

[2] A. Astaneh-Asl, Seismic design steel column-tree moment resisting frames. Berkeley, California: Structural Steel Educational Council, 1997.

[3] KBC, Korea building code and commentary. Architectural Institute of Korea, 2009.

[4] K. Lee, "Cyclic testing of steel column-tree moment connections with various beam splice lengths," Steel Compos. Struct., vol. 16, no. 2, pp. 221-231, 2014.

[5] K. Oh, "Cyclic testing of steel column-tree moment connections with weakened beam splices," Int. J. Steel Struct., vol. 14, no. 3, pp. 471-478, 2014.

[6] K. Oh, "Cyclic testing of weak-axis column-tree connections with formation of plastic hinge at beam splice," Earthq. Struct., vol. 8, no. 5, pp. 1039-1054, 2015.

[7] K. Oh, "Seismic performance evaluation of weak axis column-tree moment connections with reduced beam section," J. Constr. Steel Res., vol. 105, pp. 28-38, 2015. 